\title{
Effect of sustained virological response after direct-acting antivirals on liver fibrosis in patients with chronic HCV infection
}

Ramy H. Agwa' ${ }^{1}$, Mohamed H. Elgazzar', Islam A. El-Zayyadi', Ahmed M. Saed ${ }^{1}$, Mayada A. Ghannam² and Ahmed Saleh ${ }^{1 *}$ (D)

\begin{abstract}
Background: Direct-acting antivirals (DAAs) have revolutionized the therapy of HCV infection with higher sustained virological response (SVR) rates. Fibrosis regression after achieving SVR to DAA remains to be evaluated in chronic hepatitis $C$ patients. One of the main inquiries here is what occurs with liver fibrosis after achieving a SVR, albeit the current DAA was not intended to be antifibrotic. Liver biopsy was replaced by various non-invasive methods, like FIB4 score and fibroscan. The aim of the study was to evaluate the impact of SVR following DAAs on liver fibrosis in chronic HCV patients.
\end{abstract}

Results: Five hundred of $1170 \mathrm{~F} 4$ treated patients (42.7\%) improved and became $190 \mathrm{~F} 3,90 \mathrm{~F} 2$, and $220 \mathrm{~F} 1$. Also, 40 of $60 \mathrm{~F} 3$ patients improved and became $10 \mathrm{~F} 2$ and $30 \mathrm{~F} 1$. Also, 350 of 1230 treated patients (28.4\%) transited from significant fibrosis $(\geq F 3)$ to non-significant fibrosis $(\leq F 2)$. There was a significant improvement of FIB-4 $(p<0.001)$ in the improved group after DAAs were proved by liver stiffness measurement.

Conclusion: Treatment of chronic HCV with DAAs is associated with regression of liver fibrosis as about $28 \%$ of patients improved from significant fibrosis $(\geq F 3)$ to non-significant fibrosis $(\leq F 2)$ after treatment.

Keywords: Direct-acting antivirals, HCV, Fibrosis

\section{Background}

Hepatitis $\mathrm{C}$ virus (HCV) infection is one of the major causes of liver cirrhosis worldwide [1]. The number of individuals with chronic $\mathrm{HCV}$ all over the world is approximately 185 million [2].

In the last few years, antiviral therapy for $\mathrm{HCV}$ has been rapidly evolving with the introduction and proliferation of direct-acting antiviral (DAA) therapies. They revolutionized the therapy of HCV infection with higher efficacy and sustained virological response (SVR) rates, shortened and simplified regimens, and minimal adverse effects [3]. However, achieving sustained SVR

\footnotetext{
*Correspondence: drahmedsaleh1981@gmail.com

${ }^{1}$ Department of Internal Medicine, Hepatology \& Gastroenterology unit,

Faculty of Medicine, Mansoura University, Mansoura, Egypt

Full list of author information is available at the end of the article
}

does not instantly reverse HCV-related hepatic fibrosis or cirrhosis.

Reversion of hepatic fibrosis has been an important point of exploration and study among liver diseases experts for several decades. Previous studies on the impact of interferon-based HCV therapy on hepatic fibrosis have shown regression of fibrosis over periods of up to 48 months in some patients after therapy [4-6].

Nowadays, liver stiffness assessment using vibrationcontrolled transient elastography (VCTE) has superseded liver biopsy as a favored non-invasive modality ${ }^{7}$. Moreover, the value of TE has been assessed in checking the progression of fibrosis in the set of HCV relapses after liver transplantation [7].

Numerous noninvasive laboratory scores such as APRI, FIB-4, and FIB-5 have been verified to be precise 
in chronic liver disease staging before antiviral treatment and assessing hepatic fibrosis in HCV patients. Furthermore, they have been useful for follow-up of patients with chronic HCV and to evaluate the impact of DAA therapy $[8,9]$.

The aim of the current study was to assess the impact of SVR following DAAs on liver fibrosis in chronic $\mathrm{HCV}$ patients.

\section{Methods}

\section{Study design}

This is a prospective study that was conducted on patients attending to the Hepatology Clinics, Specialized Medical Hospital, Mansoura University, Egypt, for follow-up of chronic HCV infection during the period from January 2019 to January 2021.

\section{Patients}

Our study was conducted on 1380 chronic HCV patients; 1230 patients received DAAs and 150 patients did not receive treatment on their request (control group). We included patients who are 16 years or older with $\mathrm{HCV}$ who are a candidate for DAAs. Patients with radiologically suspected focal lesions either malignant or not, coinfection with other viruses, presence of decompensated liver cirrhosis (Child-Pugh more than 7), and organ failure were excluded from the study. Chronic $\mathrm{HCV}$ infection was diagnosed by positive RT-PCR RNA HCV \pm abnormal liver function tests and the presence of stigmata of chronic liver disease. HCV RNA was quantified by real-time PCR assay (COBAS AmpliPrep/COBAS TaqMan 48, Roche Molecular Diagnostics). HCV genotype was detected by Versant HCV genotype 2.0 assay (LiPA-Siemens, Erlangen, Germany).

\section{Patients evaluation}

Before the start of antiviral treatment, all patients were subjected to full history and clinical assessment.

\section{Laboratory assessment}

Laboratory tests were done as complete blood count, liver enzymes aspartate aminotransferase, alanine aminotransferase (ALT, AST), serum bilirubin, serum creatinine, $\alpha$ fetoprotein, and INR.

\section{Transient elastography}

The liver stiffness of the patients was assessed via Fibroscan (Echosens, Paris, France). For staging, the subsequent cutoff values were used: were F1 $(>4.8 \mathrm{kPa})$, F2 $(>8.3 \mathrm{kPa}), \mathrm{F} 3(>10.1 \mathrm{kPa})$, and F4 $(>13.4 \mathrm{kPa})$ [10]. Liver stiffness was considered as the median of all valid measurements. For high BMI ( $\geq 30 \mathrm{~kg} / \mathrm{m} 2)$, examination with the XL probe, with two experienced operators, was done.
Transient elastography was done before and immediately after treatment and after 48 weeks.

FIB-4 was calculated [age (years) $\times$ AST $(\mathrm{IU} / \mathrm{l}) /$ (platelet count $(109 / 1) \times \sqrt{ }$ ALT $(I U / 1))]$ for all patients. Fibrosis is considered to be significant if $\geq$ F3 and non-significant fibrosis if $\leq \mathrm{F} 2$. If fibrosis decreases by only one stage it is considered as fibrosis regression and stationary fibrosis if there is no change in the fibrosis stage.

All patients were evaluated by all the previous parameters before treatment and immediately after treatment, then after 12-48 weeks.

\section{Definitions}

Fibrosis regression: decrease liver fibrosis by one or more stages as F4 to $<\mathrm{F} 3$.

Fibrosis progression: increase liver fibrosis by one or more stages as F3 to $>$ F4.

Stationary course: No change in liver fibrosis stage.

\section{Treatment protocols}

At the Virology unit, Specialized Medical Hospital, Mansoura University, Egypt, all patients were evaluated for anti-HCV treatment. The DAAs regimens were used according to the guidelines of the National Committee for Control of Viral Hepatitis (NCCVH) in Egypt. We treated the patients with the following combinations: SOF plus DCV, SOF/DCV/RBV, SOF/RBV, LDV/SOF, and interferon-based.

\section{Statistical analysis and data interpretation}

Data were fed to the computer and analyzed using IBM SPSS Corp. Released 2013. IBM SPSS Statistics for Windows, Version 22.0. Armonk, NY: IBM Corp. Qualitative data were described using numbers and percentages. Quantitative data were described using median (minimum and maximum) and interquartile range for nonparametric data and mean, standard deviation for parametric data after testing normality using Kolmogorov-Smirnov test. Significance of the obtained results was judged at the 0.05 level. Chi-square test, Fisher's exact test, and Monte Carlo test were used for comparison of 2 or more groups for qualitative variables. Stewart-Maxwell test was used to compare follow-up periods for categorical variables with more than 2 categories. Student's $t$ test was used to compare 2 independent groups, paired $t$ test and repeated measures ANOVA, to compare between studied periods with post hoc Tukey test for parametric variables. Mann-Whitney $U$ test was used to compare 2 independent groups, Wilcoxon signed rank test and Friedman test were used to compare studied periods for non-parametric variables. 
Table 1 Demographic and clinical characteristics of the studied patients

\begin{tabular}{|c|c|c|}
\hline & $n=1380$ & $\%$ \\
\hline \multicolumn{3}{|l|}{ Age/years } \\
\hline Mean $\pm S D$ & $56.39 \pm 7.81(39.0-75.0)$ & \\
\hline \multicolumn{3}{|l|}{ Sex } \\
\hline Male & 630 & 45.0 \\
\hline Female & 770 & 55.0 \\
\hline DM & 380 & 27.1 \\
\hline Hypertension & 190 & 13.6 \\
\hline Associated comorbidities & 120 & 8.6 \\
\hline
\end{tabular}

\section{Results}

The current study was conducted on 1380 chronic HCV patients, with mean age of $56.39 \pm 7.81$ years, 770 females and 610 males. DM was found in $27.1 \%$ and HTN in $13.6 \%$ of patients (Table 1). The common regimen used in HCV treatment was SOF/DCV/RBV in 920 patients.

There was a significant improvement of FIB- $4(p<0.001)$ in the improved group after DAAs proved by liver stiffness measurement. Also, there was significant improvement after DAAs as regard AST, s. bilirubin, s. albumin, and creatinine $(p<0.001,0.01,0.002$, and 0.003 , respectively) (Table 2).

Table 3 compares the radiographic and laboratory results between the control group and interventional arm where there was a significant improvement in the

Table 2 Radiographic and laboratory results pre and post treatment among studied cases

\begin{tabular}{|c|c|c|c|c|c|c|c|c|}
\hline & Pre & Post 1 & Post 2 & $\begin{array}{l}\text { Overall } \\
\text { significance }\end{array}$ & 1 vs 2 & 1 vs 3 & 2 vs 3 & $\begin{array}{l}\text { Percent of } \\
\text { improvement }\end{array}$ \\
\hline Fibroscan & $22.30(8.9-75.0)$ & $17.05(2.9-75.0)$ & $15.1(2.9-56.0)$ & $p<0.001^{*}$ & $p<0.001^{*}$ & $p<0.001^{*}$ & $p<0.001^{*}$ & $\begin{array}{l}\% 1=22.7 \\
\% 2=38.9 \\
\% 3=20.92\end{array}$ \\
\hline FIB-4 & $2.41(0.82-20.68)$ & $2.19(0.58-19.36)$ & $2.06(0.58-17.64)$ & $p<0.001^{*}$ & $p=0.001^{*}$ & $p<0.001^{*}$ & $p=0.22$ & $\begin{array}{l}\% 1=16.8 \\
\% 2=17.5 \\
\% 3=0.84\end{array}$ \\
\hline ALT & $28.0(2.0-88.0)$ & $26.5(10.0-128.0)$ & $24.0(8.0-86.0)$ & $p=0.257$ & $p=0.313$ & $p=0.005^{*}$ & $p=0.032^{*}$ & $\begin{array}{l}\% 1=5.4 \\
\% 2=12.2 \\
\% 3=7.2\end{array}$ \\
\hline AST & $34.0(11.0-94.0)$ & $28.0(11.0-92.0)$ & $28.0(7.0-96.0)$ & $p<0.001^{*}$ & $p=0.009^{*}$ & $p<0.001^{*}$ & $p<0.001^{*}$ & $\begin{array}{l}\% 1=12.3 \\
\% 2=19.04 \\
\% 3=7.7\end{array}$ \\
\hline Platelet & $145.0(33.0-343.0)$ & $148.5(30.0-374.0)$ & $145.0(37.0-333.0)$ & $p=0.175$ & $p=0.117$ & $p=0.653$ & $p=0.002^{*}$ & $\begin{array}{l}\% 1=4.4 \\
\% 2=1.06 \\
\% 3=3.2\end{array}$ \\
\hline $\mathrm{Hb}$ & $13.0 \pm 1.46(9.3-17.1)$ & $\begin{array}{l}13.11 \pm 1.33 \\
(9.5-16.0)\end{array}$ & $\begin{array}{l}13.17 \pm 1.17 \\
(10.0-17.1)\end{array}$ & $p=0.131$ & $p=0.14$ & $p=0.163$ & $p=0.926$ & $\begin{array}{l}\% 1=0.8 \\
\% 2=1.3 \\
\% 3=0.46\end{array}$ \\
\hline WBCS & $5.75(1.6-15.0)$ & $5.77(2.44-13.0)$ & $6.0(2.6-13.0)$ & $p=0.398$ & $p=0.127$ & $p=0.125$ & $p=0.333$ & $\begin{array}{l}\% 1=4.1 \\
\% 2=5.6 \\
\% 3=1.3\end{array}$ \\
\hline Bilirubin & $0.90(0.3-2.5)$ & $0.89(0.30-5.0)$ & $0.90(0.3-5.0)$ & $p=0.01^{*}$ & $p=0.769$ & $p=0.102$ & $p=0.024^{*}$ & $\begin{array}{l}\% 1=5.59 \\
\% 2=7.4 \\
\% 3=1.7\end{array}$ \\
\hline Albumin & $4.135 \pm 0.41(3.0-5.0)$ & $4.09 \pm 0.48(2.5-5.5)$ & $4.05 \pm 0.38(2.8-5.4)$ & $p=0.002^{*}$ & $p=0.174$ & $p=0.005^{*}$ & $p=0.06$ & $\begin{array}{l}\% 1=1.1 \\
\% 2=2.1 \\
\% 3=0.98\end{array}$ \\
\hline Creatinine & $0.9(0.21-1.6)$ & $1.0(0.4-2.2)$ & $1.0(0.6-1.4)$ & $p=0.003^{*}$ & $p=0.02^{*}$ & $p<0.001^{*}$ & $p=0.195$ & $\begin{array}{l}\% 1=5.0 \\
\% 2=6.7 \\
\% 3=1.6\end{array}$ \\
\hline $\begin{array}{l}\text { Alkaline phos- } \\
\text { phatase }\end{array}$ & $116.0(106-146)$ & $93.5(65.0-245.0)$ & $127.5(111.0-145.0)$ & $p=0.001^{*}$ & $p=0.02^{*}$ & $p=0.001^{*}$ & $p=0.01^{*}$ & $\begin{array}{l}\% 1=1.3 \\
\% 2=4.1 \\
\% 3=2.82\end{array}$ \\
\hline EGD & $n=18$ & $N=9$ & $N=6$ & $p=0.717$ & $p=0.102$ & $p=0.564$ & $p=0.317$ & \\
\hline OVs & $5(27.8)$ & $1(11.1)$ & $0(0.0)$ & & & & & \\
\hline PHG & $3(16.7)$ & $7(77.8)$ & $6(100.0)$ & & & & & \\
\hline Both & $7(38.9)$ & $1(11.1)$ & $0(0.0)$ & & & & & \\
\hline Free & $3(16.7)$ & $0(0.0)$ & $0(0.0)$ & & & & & \\
\hline
\end{tabular}

Used tests: Friedman test, repeated measures ANOVA test with post hoc Tukey test, Wilcoxon signed rank test, and Stewart Maxwell test. Parameters described as mean \pm SD, median (range) (interquartile range), number and percentage \%1: percent of improvement between pre and post 1 follow-up, \%2: percent of improvement between pre and post 2,\%3: percent of improvement between post 1 and post 2 
Table 3 Comparison of radiographic and laboratory results between control and interventional arm

\begin{tabular}{|c|c|c|c|}
\hline & $\begin{array}{l}\text { Control arm (untreated } \\
\text { cases) }\end{array}$ & $\begin{array}{l}\text { Intervention arm (treated } \\
\text { cases) }\end{array}$ & Test of significance \\
\hline \multicolumn{4}{|l|}{ Fibroscan } \\
\hline 1 & $27.0(15.0-73.0)$ & $21.6(8.9-75.0)$ & $P=0.167$ \\
\hline 2 & $21.3(12.5-175.0)$ & $16.8(2.9-68.3)$ & $P=0.07$ \\
\hline 3 & $17.40(10.3-27.0)$ & $14.3(2.9-56.0)$ & $P=0.111$ \\
\hline Test of significance between pre and post treatment & $\begin{array}{l}P 1=0.05 \\
P 2=0.005^{*} \\
P 3=0.001^{*}\end{array}$ & $\begin{array}{l}P 1=0.001^{*} \\
P 2=0.001^{*} \\
P 3=0.001^{*}\end{array}$ & \\
\hline \multicolumn{4}{|l|}{ FIB-4 } \\
\hline 1 & $2.76(0.93-9.030$ & $2.38(0.82-20.68)$ & $P=0.511$ \\
\hline 2 & $2.01(0.58-8.74)$ & $2.289(0.58-16.39)$ & $P=0.949$ \\
\hline 3 & $2.10(0.74-6.89)$ & $2.065(0.58-17.64)$ & $P=0.956$ \\
\hline Test of significance between pre and post treatment & $\begin{array}{l}P 1=0.156 \\
P 2=0.019^{*} \\
P 3=0.221\end{array}$ & $\begin{array}{l}P 1=0.003^{*} \\
P 2=0.002^{*} \\
P 3=0.383\end{array}$ & \\
\hline \multicolumn{4}{|l|}{ ALT } \\
\hline 1 & $32.0(15.0-88.0)$ & $27.0(2.0-78.0)$ & $P=0.08$ \\
\hline 2 & $28.0(15.0-59.0)$ & $26.0(10.0-128.0)$ & $P=0.272$ \\
\hline 3 & $28.0(18.0-43.00$ & $24.0(8.0-86.0)$ & $P=0.059$ \\
\hline Test of significance between pre and post treatment & $\begin{array}{l}P 1=0.271 \\
P 2=0.362 \\
P 3=0.969\end{array}$ & $\begin{array}{l}P 1=0.507 \\
P 2=0.009^{*} \\
P 3=0.026^{*}\end{array}$ & \\
\hline \multicolumn{4}{|l|}{ AST } \\
\hline 1 & $38(17-94)$ & $34(11-85)$ & $P=0.233$ \\
\hline 2 & $32(17-79)$ & $28(11-92)$ & $P=0.143$ \\
\hline 3 & $28.5(20-45)$ & $27(7-96)$ & $P=0.690$ \\
\hline Test of significance between pre and post treatment & $\begin{array}{l}P 1=0.300 \\
P 2=0.064 \\
P 3=0.02^{*}\end{array}$ & $\begin{array}{l}P 1=0.019^{*} \\
P 2<0.001^{*} \\
P 3=0.003^{*}\end{array}$ & \\
\hline \multicolumn{4}{|l|}{ Platelets } \\
\hline 1 & $147(57-259)$ & $145(33-343)$ & $P=0.954$ \\
\hline 2 & $154(61-355)$ & $148(30-374)$ & $P=0.347$ \\
\hline 3 & $146(65-280)$ & $145(37-333)$ & $P=0.639$ \\
\hline Test of significance between pre and post treatment & $\begin{array}{l}P 1=0.101 \\
P 2=0.414 \\
P 3=0.014^{*}\end{array}$ & $\begin{array}{l}\mathrm{P} 1=0.275 \\
\mathrm{P} 2=0.844 \\
\mathrm{P} 3=0.021^{*}\end{array}$ & \\
\hline \multicolumn{4}{|l|}{ Bilirubin } \\
\hline 1 & $0.90(0.3-2.0)$ & $0.90(0.30-2.50$ & $P=0.734$ \\
\hline 2 & $0.80(0.5-1.4)$ & $0.90(0.3-5.0)$ & $P=0.539$ \\
\hline 3 & $0.85(0.7-2.5)$ & $0.90(0.3-5.0)$ & $P=0.701$ \\
\hline Test of significance between pre and post treatment & $\begin{array}{l}P 1=0.798 \\
P 2=0.306 \\
P 3=0.107\end{array}$ & $\begin{array}{l}P 1=0.726 \\
P 2=0.169 \\
P 3=0.061\end{array}$ & \\
\hline \multicolumn{4}{|l|}{ Albumin } \\
\hline 1 & $3.93 \pm 0.53$ & $4.16 \pm 0.39$ & $P=0.04^{*}$ \\
\hline 2 & $4.19 \pm 0.48$ & $4.08 \pm 0.49$ & $P=0.408$ \\
\hline 3 & $4.09 \pm 0.33$ & $4.05 \pm 0.39$ & $P=0.718$ \\
\hline Test of significance between pre and post treatment & $\begin{array}{l}P 1=0.133 \\
P 2=0.606 \\
P 3=0.123\end{array}$ & $\begin{array}{l}P 1=0.096 \\
P 2=0.007^{*} \\
P 3=0.365\end{array}$ & \\
\hline \multicolumn{4}{|l|}{ EGD1 } \\
\hline OVs & 0 & $50(4.0)$ & $P=0.645$ \\
\hline PHG & 0 & $30(2.4)$ & \\
\hline
\end{tabular}


Table 3 (continued)

\begin{tabular}{|c|c|c|c|}
\hline & $\begin{array}{l}\text { Control arm (untreated } \\
\text { cases) }\end{array}$ & $\begin{array}{l}\text { Intervention arm (treated } \\
\text { cases) }\end{array}$ & Test of significance \\
\hline Both & $10(6.7)$ & $60(4.8)$ & \\
\hline Free & 0 & $30(2.4)$ & \\
\hline \multicolumn{4}{|l|}{ EGD2 } \\
\hline OVs & 0 & $10(0.8)$ & $P=0.133$ \\
\hline PHG & $10(6.7)$ & $60(4.8)$ & \\
\hline Both & $10(6.7)$ & $0(0.0)$ & \\
\hline Free & 0 & $0(0.0)$ & \\
\hline \multicolumn{4}{|l|}{ EGD3 } \\
\hline PHG & $20(13.3)$ & $40(3.2)$ & $P=0.125$ \\
\hline Test of significance between pre and post treatment & $\begin{array}{l}P 1=0.317 \\
P 2=1.0 \\
P 3=0.317\end{array}$ & $\begin{array}{l}P 1=0.180 \\
P 2=1.0 \\
P 3=1.0\end{array}$ & \\
\hline
\end{tabular}

Used tests: Friedman test, repeated measures ANOVA test with post hoc Tukey test, Wilcoxon signed rank test, and Stewart Maxwell test

Parameters described as mean $\pm S D$, median (range) (interquartile range) \%1: percent of improvement between pre and post 1 follow-up, \%2; percent of improvement between pre and post 2, \%3: percent of improvement between post 1 and post 2

* Statistically significant

radiographic, laboratory, and endoscopic parameters of the treated patients.

The study included 150 cirrhotic patients (F4 by fibroscan), treated group (1170 F4 and 6 F3). After treatment, 500 of 1170 F4 patients (42.7\%) improved and became 190 F3, 90 F2, and 220 F1. Also, 40 of 60 F3 patients improved and became $10 \mathrm{~F} 2$ and $30 \mathrm{~F} 1$. After treatment 350 of 1230 treated patients (28.4\%) transited from significant fibrosis $(\geq F 3)$ to non-significant fibrosis $(\leq F 2)$. Moreover, 840 of 1230 treated patients (68.3\%) showed a reduction in liver stiffness $\geq 30 \%$ from their baseline by fibroscan.

In Table 4, we compared the radiographic and laboratory results between controls, improved, and nonimproved cases. We found that non-improved cases were older, with a high incidence of DM, and have lower baseline ALT, AST, serum albumin, platelets, and high bilirubin than the improved cases.

\section{Discussion}

$\mathrm{HCV}$ is a worldwide prevalent virus and a major cause of death and morbidity. Introduction of DAAs has resulted in a substantial breakthrough in HCV treatments as they can achieve SVR in almost $100 \%$ of patients with chronic hepatitis C; however, the ultimate goal of $\mathrm{HCV}$ treatments is to prevent cirrhosis development and its sequels [11].

Our study was conducted on 1380 chronic HCV patients with mean age $56.39 \pm 7.81$ years, 770 females and 610 males. The common regimen used in HCV treatment was SOF/DCV/RBV (920 patients).
In our study, 500 of $1170 \mathrm{~F} 4$ treated patients $(42.7 \%)$ improved and became 190 F3, 90 F2, and 220 F1. Also, 40 of $60 \mathrm{~F} 3$ patients improved and became $10 \mathrm{~F} 2$ and $30 \mathrm{~F} 1$. After treatment 350 of the treated patients (28.4\%) transited from significant fibrosis $(\geq F$ 3) to non-significant fibrosis $(\leq \mathrm{F} 2)$. Also, There was a significant improvement of FIB-4 $(p<0.001)$ in the improved group after DAAs proved by liver stiffness measurement.

Similar results were observed by Pietsch et al. [12] who evaluated liver stiffness changes (for 96 weeks) using transient elastography in only 143 chronic $\mathrm{HCV}$ patients with achieved SVR following DAAs. There was a significant overall long-term improvement of liver stiffness values in all patients in their study although they have not clarified the median of transient elastography changes. Interestingly, in another study of 260 patients with chronic HCV, Lledó et al. reported that $40 \%$ of patients showed significant fibrosis regression. Furthermore, this fibrosis regression was more obvious in patients who have advanced fibrosis at baseline [13].

Also, a systematic review and meta-analysis by Singh et al revised 11 studies evaluating LSM before and after DAAs demonstrated a rapid regression in liver stiffness during DAAs treatment and a slower but steady regression after treatment as LSM declined by $2.4 \mathrm{kPa}$ at the end of treatment, by $3.1 \mathrm{kPa} 4-24$ weeks after treatment, by $3.2 \mathrm{kPa} 24-48$ weeks after treatment, and by $4.1 \mathrm{kPa}>12$ months after treatment. The pooled regression in liver fibrosis in these studies may be related to the design of these studies as the majority of those studies were retrospective and included a small number of patients with advanced fibrosis and cirrhosis [14]. 
Table 4 Comparison of radiographic and laboratory results between controls, improved and non-improved cases

\begin{tabular}{|c|c|c|c|c|c|}
\hline & Control arm $n=150$ & $\begin{array}{l}\text { Non-improved } \\
\text { cases } n=880\end{array}$ & Improved cases $n=350$ & Test of significance & Test of sig. \\
\hline Age/years & $58.33 \pm 8.16$ & $57.14 \pm 6.88$ & $53.43 \pm 9.33$ & $\begin{array}{l}F=3.47 \\
P=0.034^{*}\end{array}$ & $\begin{array}{l}\mathrm{P} 1=0.579 \\
\mathrm{P} 2=0.041^{*} \\
\mathrm{P} 3=0.017^{*}\end{array}$ \\
\hline \multicolumn{6}{|l|}{ Sex } \\
\hline Male & $60(40.0)$ & $340(38.6)$ & $210(60.0)$ & $x^{2}=4.75$ & $\mathrm{p} 1=0.92$ \\
\hline Female & $90(60.0)$ & $540(61.4)$ & $140(40.0)$ & $P=0.093$ & $\begin{array}{l}p 2=0.19 \\
p 3=0.032^{*}\end{array}$ \\
\hline $\mathrm{DM}$ & $30(20.0)$ & $310(35.2)$ & $40(11.4)$ & $M C P=0.023^{*}$ & $\begin{array}{l}\mathrm{p} 1=0.246 \\
\mathrm{p} 2=0.423 \\
\mathrm{p} 3=0.008^{*}\end{array}$ \\
\hline Hypertension & $30(20.0)$ & $130(14.8)$ & $30(8.6)$ & $M C P=0.506$ & $\begin{array}{l}\mathrm{p} 1=0.699 \\
\mathrm{p} 2=0.348 \\
\mathrm{p} 3=0.356\end{array}$ \\
\hline \multicolumn{6}{|c|}{ Associated comorbidities } \\
\hline$-V E$ & $20(13.3)$ & $80(9.1)$ & $20(5.7)$ & $M C P=0.665$ & $\mathrm{p} 1=0.608$ \\
\hline$+\mathrm{VE}$ & $130(86.7)$ & $800(90.9)$ & $330(94.3)$ & & $\begin{array}{l}\mathrm{p} 2=0.574 \\
\mathrm{p} 3=0.723\end{array}$ \\
\hline \multicolumn{6}{|l|}{ Spleen US } \\
\hline 1 & $13.57 \pm 3.19$ & $13.63 \pm 2.39$ & $12.37 \pm 1.61$ & $F=3.74, P=0.026^{*}$ & $\mathrm{p} 1=0.92, \mathrm{p} 2=0.09, \mathrm{p} 3=0.008^{*}$ \\
\hline 2 & $13.03 \pm 2.75$ & $13.40 \pm 2.33$ & $12.09 \pm 1.50$ & $F=4.45, P=0.013^{*}$ & $\mathrm{p} 1=0.55, \mathrm{p} 2=0.16, \mathrm{p} 3=0.003^{*}$ \\
\hline 3 & $12.75 \pm 2.14$ & $13.21 \pm 1.31$ & $11.91 \pm 1.31$ & $F=5.74, P=0.004^{*}$ & $\mathrm{p} 1=0.41, \mathrm{p} 2=0.17, \mathrm{p} 3=0.001^{*}$ \\
\hline \multicolumn{6}{|l|}{ Portal vein US } \\
\hline 1 & $12.40 \pm 2.92$ & $12.81 \pm 2.44$ & $10.40 \pm 1.75$ & $F=13.29, P<0.001^{*}$ & $\mathrm{p} 1=0.54, \mathrm{p} 2=0.006^{*}, \mathrm{p} 3<0.001^{*}$ \\
\hline 2 & $12.13 \pm 2.79$ & $12.58 \pm 2.41$ & $10.09 \pm 1.46$ & $F=15.42, P=0.001^{*}$ & $\mathrm{p} 1=0.48, \mathrm{p} 2=0.004^{*}, \mathrm{p} 3=0.001^{*}$ \\
\hline 3 & $11.50 \pm 2.28$ & $12.35 \pm 2.42$ & $9.89 \pm 1.25$ & $F=16.1, P=0.001^{*}$ & $\mathrm{p} 1=0.17, \mathrm{p} 2=0.02^{*}, \mathrm{p} 3=0.001^{*}$ \\
\hline \multicolumn{6}{|l|}{ ALT } \\
\hline 1 & $32(15-88)$ & $24.5(6-60)$ & $34(2-78)$ & $\mathrm{KW}, P=0.001^{*}$ & $\mathrm{p} 1=01^{*}, \mathrm{p} 2=0.82, \mathrm{p} 3<0.001^{*}$ \\
\hline 2 & $28(15-59)$ & $25(10-128)$ & $28(16-46)$ & $\mathrm{KW}, P=0.23$ & $p 1=0.17, p 2=0.65, p 3=0.20$ \\
\hline 3 & $28(18-43)$ & $24(8-86)$ & $26(10-43)$ & $K W, P=0.09$ & $\mathrm{p} 1=0.05, \mathrm{p} 2=0.22, \mathrm{p} 3=0.25$ \\
\hline \multicolumn{6}{|l|}{ AST } \\
\hline 1 & $38(17-94)$ & $34(11-85)$ & $38(19-71)$ & $\mathrm{KW}, P=0.04^{*}$ & $\mathrm{p} 1=0.14, \mathrm{p} 2=0.92, \mathrm{p} 3=0.02^{*}$ \\
\hline 2 & $32(17-79)$ & $28(11-92)$ & $26(13-62)$ & $K W, P=0.057$ & $\mathrm{p} 1=0.27, \mathrm{p} 2=0.025^{*}, \mathrm{p} 3=0.07$ \\
\hline 3 & $28.5(20-45)$ & $28.5(7-96)$ & $23(14-45)$ & $\mathrm{KW}, P=0.003^{*}$ & $\mathrm{p} 1=0.61, \mathrm{p} 2=0.024^{*}, \mathrm{p} 3=0.001^{*}$ \\
\hline \multicolumn{6}{|l|}{ Platelet } \\
\hline 1 & $147(57-259)$ & $126.5(33-256)$ & $196(60-343)$ & $\mathrm{KW}, P=0.001^{*}$ & $p 1=0.47, p 2=0.07, p 3=0.001^{*}$ \\
\hline 2 & $154(61-355)$ & $138.5(30-344)$ & $187(72-374)$ & $\mathrm{KW}, P=0.001^{*}$ & $\mathrm{p} 1=0.12, \mathrm{p} 2=0.53, \mathrm{p} 3<0.001^{*}$ \\
\hline 3 & $138.5(30-344)$ & $134(37-259)$ & $184(60-333)$ & $\mathrm{KW}, P=0.001^{*}$ & $\mathrm{p} 1=0.24, \mathrm{p} 2=0.19, \mathrm{p} 3<0.001^{*}$ \\
\hline \multicolumn{6}{|l|}{$\mathrm{Hb}$} \\
\hline 1 & $13.45 \pm 1.19$ & $12.8 \pm 1.58$ & $13.11 \pm 1.27$ & $F=1.08, P=0.342$ & $\mathrm{p} 1=.17, \mathrm{p} 2=0.46, \mathrm{p} 3=0.44$ \\
\hline 2 & $13.20 \pm 0.95$ & $13.05 \pm 1.40$ & $13.20 \pm 1.31$ & $F=0.21, P=0.81$ & $\mathrm{p} 1=0.682, \mathrm{p} 2=0.99, \mathrm{p} 3=0.563$ \\
\hline 3 & $12.79 \pm 0.79$ & $13.15 \pm 1.19$ & $13.29 \pm 1.22$ & $F=0.92, P=0.40$ & $p 1=0.286, p 2=0.17, p 3=0.54$ \\
\hline \multicolumn{6}{|l|}{ WBCS } \\
\hline 1 & $7.62 \pm 2.14$ & $5.85 \pm 2.35$ & $6.61 \pm 2.61$ & $F=4.06, P=0.019^{*}$ & $\mathrm{p} 1=0.009^{*}, \mathrm{p} 2=0.17, \mathrm{p} 3=0.11$ \\
\hline 2 & $6.83 \pm 2.05$ & $5.78 \pm 1.94$ & $6.08 \pm 2.32$ & $F=1.74, P=0.179$ & $p 1=0.07, p 2=0.24, p 3=0.47$ \\
\hline 3 & $6.04 \pm 1.39$ & $5.81 \pm 1.72$ & $6.03 \pm 2.06$ & $F=0.232, P=0.793$ & $\mathrm{p} 1=0.66, \mathrm{p} 2=0.98, \mathrm{p} 3=0.55$ \\
\hline \multicolumn{6}{|l|}{ Bilirubin } \\
\hline 1 & $0.887 \pm 0.43$ & $0.931 \pm 0.441$ & $0.902 \pm 0.34$ & $F=0.11, P=0.896$ & $p 1=0.70, p 2=0.90, p 3=0.73$ \\
\hline 2 & $0.931 \pm 0.44$ & $0.902 \pm 0.34$ & $1.05 \pm 1.0$ & $F=0.59, P=0.55$ & $p 1=0.50, p 2=0.29, p 3=0.48$ \\
\hline 3 & $0.96 \pm 0.45$ & $1.04 \pm 0.61$ & $0.860 \pm 0.21$ & $F=1.53, P=0.22$ & $\mathrm{p} 1=0.61, \mathrm{p} 2=0.52, \mathrm{p} 3=0.08$ \\
\hline \multicolumn{6}{|l|}{ Albumin } \\
\hline 1 & $3.93 \pm 0.53$ & $4.13 \pm 0.39$ & $4.22 \pm 0.37$ & $F=2.54, P=0.082$ & $\mathrm{p} 1=0.08, \mathrm{p} 2=0.03^{*}, \mathrm{p} 3=0.31$ \\
\hline
\end{tabular}


Table 4 (continued)

\begin{tabular}{|c|c|c|c|c|c|}
\hline & Control arm $n=150$ & $\begin{array}{l}\text { Non-improved } \\
\text { cases } n=880\end{array}$ & Improved cases $n=350$ & Test of significance & Test of sig. \\
\hline 2 & $4.19 \pm 0.48$ & $4.12 \pm 0.50$ & $3.99 \pm 0.43$ & $F=1.24, P=0.29$ & $\mathrm{p} 1=0.59, \mathrm{p} 2=0.18, \mathrm{p} 3=0.18$ \\
\hline 3 & $4.09 \pm 0.33$ & $4.03 \pm 0.41$ & $4.07 \pm 0.26$ & \multirow[t]{2}{*}{$F=0.198, P=0.82$} & \multirow[t]{2}{*}{$\mathrm{p} 1=0.62, \mathrm{p} 2=0.88, \mathrm{p} 3=0.63$} \\
\hline EGD1 & $N=10$ & $N=160$ & $N=10$ & & \\
\hline OVs & $0(0.0)$ & $50(31.2)$ & $0(0.0)$ & \multirow[t]{4}{*}{$M C P=0.334$} & \multirow{5}{*}{$\begin{array}{l}\mathrm{p} 1=0.678 \\
\mathrm{p} 2=0.157 \\
\mathrm{p} 3=0.175\end{array}$} \\
\hline PHG & $0(0.0)$ & $20(12.5)$ & $10(100)$ & & \\
\hline Both & $10(100)$ & $60(37.5)$ & $0(0.0)$ & & \\
\hline Free & $0(0.0)$ & $30(18.8)$ & $0(0.0)$ & & \\
\hline EGD2 & $N=20$ & $N=70$ & & & \\
\hline OVs & $0(0.0)$ & $10(14.3)$ & 0 & \multirow[t]{3}{*}{$\mathrm{MC} P=0.133$} & \multirow{3}{*}{$\begin{array}{l}\mathrm{p} 1=0.133 \\
\mathrm{p} 2=. . \\
\mathrm{p} 3=\ldots\end{array}$} \\
\hline $\mathrm{PHG}$ & $10(50.0)$ & $60(85.7)$ & 0 & & \\
\hline Both & $10(50.0)$ & $0(0.0)$ & 0 & & \\
\hline \multicolumn{6}{|l|}{ EGD3 } \\
\hline PHG & $20(100.0)$ & $40(100.0)$ & $0(0.0)$ & & \\
\hline
\end{tabular}

The chance for reversion of liver fibrosis is more pronounced in patients with early cirrhosis because the persistence of necro-inflammatory activity in milder forms of chronic liver disease may take up to $15-30$ years before achieving real significant degrees of scarring in the liver [15]. Reversion of early fibrosis is easier than older fibrosis, which becomes more stabilized progressively via collagen crosslinking by tissue transglutaminase that makes fibers less sensitive to degrading enzymes [16]. Septal fibrosis in early-stage concomitant with earlier stages of neo-angiogenesis; is more easily broken down due to its lower degree of maturity of the vessels [17].

Moreover, 840 of the treated patients (68.3\%) in our study showed a reduction in liver stiffness $\geq 30 \%$ from their baseline by fibroscan. Whereas, Chan and colleagues [18] found that only 34 of 70 patients (48.6\%) met $30 \%$ improvement in liver stiffness measurement score.

Our study also showed that regression of LSM using transient elastography was also evident by improvement of FIB-4 score. Similarly, several previous studies have shown this concordance between the regression of LSM by elastography and improvement of FIB-4 score in chronic HCV patients after DAA therapy [19-21]. From these findings, we could conclude that the combination of these non-invasive scores with transient elastography could be a good tool for the assessment of liver fibrosis.

Huang et al. [22] analyzed 40 paired liver biopsies of chronic hepatitis $\mathrm{C}$ patients before and after DAAs therapy and demonstrated significant improvement of liver inflammation and fibrosis after SVR. Furthermore, LSM by transient elastography, APRI, and FIB-4 declined significantly after SVR and predicted fibrosis well even after SVR by DAAs.

In our study, we found that non-improved cases were older, with a high incidence of DM, have lower baseline ALT, AST, serum albumin, platelets, and high bilirubin than the improved cases. Liver fibrosis is an active process comprising chronic stimulation of wound healing reaction as a response to repeated liver injury, leading to fibrillar extracellular matrix deposition in the liver and finally, liver cirrhosis if the cause of injury is not removed. Adipokines have been demonstrated to be incorporated in different obesity-related diseases, such as type 2 diabetes, hypertension, and atherosclerosis. Gathering data reveal that insulin resistance and obesity are linked to the more severe and quicker progression of the fibrosis in different chronic liver diseases [23]. In addition to low baseline ALT, AST before treatment indicates a low process of necroinflammation and the presence of significant fibrosis.

Our study may be limited by the lack of concomitant histopathological examination through liver biopsy; however, according to the American Association For Study of Liver Diseases (AASLD), the standard liver biopsy had been replaced by the non-invasive testing for liver fibrosis stages evaluation. Both transient elastography and non-invasive markers showed a high diagnostic value and their combination could be used to diagnose or exclude cirrhosis [24].

\section{Conclusions}

We concluded that treatment of chronic $\mathrm{HCV}$ with DAAs is associated with regression of liver fibrosis as about $28 \%$ of patients improved from significant fibrosis $(\geq F 3)$ to 
non-significant fibrosis $(\leq \mathrm{F} 2)$ after treatment and about $68 \%$ showed a reduction in $\mathrm{LSM}>30 \%$ from their baseline.

\section{Abbreviations}

HCV: Hepatitis C virus; DAAs: Direct-acting antiviral agents; SVR: Sustained virologic response; VCTE: Vibration controlled transient elastography; AST: Aspartate aminotransferase; ALT: Alanine aminotransferase; NCCVH: National Committee for Control of Viral Hepatitis; RBV: Ribavirin; SOF: Sofosbuvir; LDV: Ledipasvir; DCV: Daclatasvir; AASLD: American Association For Study of Liver Diseases.

\section{Acknowledgements}

We are thankful to our colleagues in the hepato-gastroenterology and hematology departments, Mansoura University. We are also thankful for our patients for their contribution and trust.

\section{Authors' contributions}

The protocol of the study, study design, methodology, follow-up of the patients, collecting data, data analysis, and writing - original draft preparation: RA, ME, and IE; Lab investigation: MG; Writing - review and editing: AS and AS. Supervision: AS. The authors read and approved the final manuscript.

\section{Funding}

This research did not receive any specific grant from funding agencies in the public, commercial, or not-for-profit sectors.

\section{Availability of data and materials}

The data analyzed during the study are included in this published article are readily available with the corresponding author for share on reasonable request.

\section{Declarations}

\section{Ethics approval and consent to participate}

The study has been approved by the ethics committee of our university (Mansoura Faculty of Medicine, Mansoura University, Institutional Review Board and has been performed following the ethical standards as laid down in the 1964 Declaration of Helsinki and its later amendments or comparable ethical standards. Informed written consent was obtained from all individual participants included in the study. Code number: R.21.03.1252.

\section{Consent for publication}

NA.

\section{Competing interests}

The authors declare that they have no competing interests.

\section{Author details}

${ }^{1}$ Department of Internal Medicine, Hepatology \& Gastroenterology unit, Faculty of Medicine, Mansoura University, Mansoura, Egypt. ${ }^{2}$ Department of Clinical Pathology, Haematology Unit, Faculty of Medicine, Mansoura University, Mansoura, Egypt.

Received: 14 July 2021 Accepted: 15 January 2022

Published online: 11 February 2022

\section{References}

1. Lavanchy D (2011) Evolving epidemiology of hepatitis C virus. Clin Microbiol Infect 17(2):107-115

2. Messina JP, Humphreys I, Flaxman A et al (2015) Global distribution and prevalence of hepatitis C virus genotypes. Hepatology 61(1):77-87

3. Kwon JH, Yoo SH, Nam SW et al (2019) Clinical outcomes after the introduction of direct antiviral agents for patients infected with genotype $1 \mathrm{~b}$ hepatitis $C$ virus depending on the regimens: A multicenter study in Korea. J Med Virol 91(6):1104-1111
4. Stasi C, Arena U, Zignego AL et al (2013) Longitudinal assessment of liver stiffness in patients undergoing antiviral treatment for hepatitis C. Dig Liver Dis 45(10):840-843

5. Martinez SM, Foucher J, Combis J-M et al (2012) Longitudinal liver stiffness assessment in patients with chronic hepatitis $C$ undergoing antiviral therapy. PLoS One 7(10):e47715-e

6. Kim JH, Kim MN, Han K-H, Kim SU (2014) Clinical application of transient elastography in patients with chronic viral hepatitis receiving antiviral treatment. Liver Int 35(4):1103-1115

7. Carrión JA, Torres F, Crespo G et al (2009) Liver stiffness identifies two different patterns of fibrosis progression in patients with hepatitis $C$ virus recurrence after liver transplantation. Hepatology 51(1):23-34

8. Bonnard P, Elsharkawy A, Zalata K et al (2014) Comparison of liver biopsy and noninvasive techniques for liver fibrosis assessment in patients infected with HCV-genotype 4 in Egypt. J Viral Hepat 22(3):245-253

9. Yosry A, Fouad R, Alem SA et al (2016) FibroScan, APRI, FIB4, and GUCI: Role in prediction of fibrosis and response to therapy in Egyptian patients with HCV infection. Arab J Gastroenterol 17(2):78-83

10. Abdelsameea E, Alsebaey A, Abdel-Razek W et al (2020) Elastography and serum markers of fibrosis versus liver biopsy in 1270 Egyptian patients with hepatitis C. Eur J Gastroenterol Hepatol 32(12):1553-1558

11. Enomoto M, Kawada N (2020) The Moral of Hepatic Fibrosis: Don't Always Believe Noninvasive Fibrosis Measurements. Dig Dis Sci 65(5):1293-1295

12. Pietsch $V$, Deterding $K$, Attia D et al (2018) Long-term changes in liver elasticity in hepatitis $C$ virus-infected patients with sustained virologic response after treatment with direct-acting antivirals. United European Gastroenterol J 6(8):1188-1198

13. Lledó GM, Carrasco I, Benítez-Gutiérrez LM et al (2018) Regression of liver fibrosis after curing chronic hepatitis $C$ with oral antivirals in patients with and without HIV coinfection. AIDS 32(16):2347-2352

14. Singh S, Facciorusso A, Loomba R, Falck-Ytter YTJCG, Hepatology. (2018) Magnitude and kinetics of decrease in liver stiffness after antiviral therapy in patients with chronic hepatitis C: a systematic review and meta-analysis. Clin Gastroenterol Hepatol 16(1):27-38 e4

15. Pinzani M (2004) Therapies for hepatic fibrosis: real hope or just academic exercise? Dig Liver Dis 36(11):714-716

16. Friedman SL (2003) Liver fibrosis-from bench to bedside. J Hepatol 38:38-53

17. Carmeliet $P$ (2003) Angiogenesis in health and disease. Nat Med 9(6):653-660

18. Chan J, Gogela N, Zheng H et al (2018) Direct-acting antiviral therapy for chronic HCV infection results in liver stiffness regression over 12 months post-treatment. Dig Dis Sci 63(2):486-492

19. Bruno G, Dell'Acqua R, Milano E, Fabrizio C, Milella MJH (2016) Early regression of liver fibrosis in HCV infected patients with or without HIV infection after treatment with DAAs. Hepatology 64:326A

20. Chen S-H, Lai H-C, Chiang I-P et al (2018) Changes in liver stiffness measurement using acoustic radiation force impulse elastography after antiviral therapy in patients with chronic hepatitis C. PLoS One 13(1):e0190455

21. Shiha G, Soliman R, Mikhail N, Ibrahim A, Serwah AH, Khattab MJG (2020) Changes in hepatic fibrosis stages after achieving SVR following directacting anti-viral treatment: a prospective study. GastroHep 2(1):39-48

22. Huang R, Rao H, Yang M et al (2020) Noninvasive Measurements Predict Liver Fibrosis Well in Hepatitis C Virus Patients After Direct-Acting Antiviral Therapy. Dig Dis Sci 65(5):1491-1500

23. Bertolani C, Marra F (2008) The role of adipokines in liver fibrosis. Pathophysiology 15(2):91-101

24. Connolly JC, Lim JK (2019) Non-invasive Fibrosis Assessment of Patients with Hepatitis C: Application of Society Guidelines to Clinical Practice. Curr Hepatol Rep 18(2):249-258

\section{Publisher's Note}

Springer Nature remains neutral with regard to jurisdictional claims in published maps and institutional affiliations. 